\title{
Gepulstes Cyclophosphamid bei Pemphigus
}

Der Standard in der Therapie des seltenen Pemphigus sind hochdosierte orale Glukokortikoide mit oder ohne Immunsuppressiva. Manche Patienten sprechen jedoch auf die üblichen Kombinationen nicht an.

D ie Arbeitsgruppe um Richard Groves vom St. Thomas Hospital in London gab 21 Pemphigus-Patienten, die nicht auf Prednisolon und Azathioprin oder Mycophenolat ansprachen, eine Therapie mit Methylprednisolon (drei Tage lang täglich $1 \mathrm{~g}$ ) und Cyclophosphamid (500 $\mathrm{mg}$ am 2 . Tag) in monatlichen Intervallen. Es gibt Hinweise, dass in monatlichen intravenösen Pulsen verabreichtes Cyclophosphamid weniger toxisch ist. In die Auswertung gingen auch die Antikörpertiter der Patienten ein, darunter die Antikörper Desmoglein 1 und 3.

Von den 21 Patienten sprachen sieben sehr gut auf die Therapie an, zwei gut, fünf mittelmäßig, sechs nur minimal und ein Patient gar nicht. Vier Patienten erreichten nach 11 bis 22 Pulsen eine komplette klinische Remission. Die Menge an Autoantikörpern nahm im Verlauf eines Jahres signifikant ab. Alle Patienten konnten ihre Prednisolon-Dosis signifikant um im Median 66\% senken. Die häufigsten Nebenwirkungen waren eine vorrübergehende Lymphopenie (12 Patienten), eine nicht lebensbedrohliche Sepsis (sieben Patienten) und frühzeitige Menopause (zwei Patientinnen).

Fazit: Eine gepulste Therapie mit Methylprednisolon und Cyclophosphamid ist eine wirksame Option bei therapierefraktären Pemphigus-Patienten. Allerdings sollten die Nebenwirkungen vor der Therapie sorgfältig abgewogen und während der Behandlung engmaschig überwacht werden. $\quad$ fah

Saha M et al. Pulsed intravenous cyclophosphamide and methylprednisolone therapy in refractory pemphigus. Br J Dermatol 18. November 2009 [Epub ahead of print]

\section{Heim-UV-B-Therapie rechnet sich}

In der holländischen multizentrischen PLUTO-Studie wurden erstmals die Kosten für eine ambulante UV-B-Phototherapie mit den entsprechenden Kosten einer UV-B-Therapie mit Heimgeräten verglichen.

$\mathrm{V}$ erglichen wurden direkte (Arzt, Behandlung etc.) und indirekte (Arbeitsausfall, Fahrtkosten etc.) gesellschaftliche Gesamtkosten einer UV-BTherapie, die Patienten entweder ambulant oder zu Hause durchführten zu zwei Zeitpunkten: a) nach Abschluss der Bestrahlungen (im Mittel nach 17,6 Wochen, $\mathrm{N}=196$ Patienten) und b) ein Jahr nach Abschluss der Phototherapie (im Mittel nach 68,4 Wochen, $\mathrm{N}=105 \mathrm{~Pa}$ tienten).

Die Gesamtkosten beliefen sich zum ersten Zeitpunkt auf $801 €$ für die Heimbehandlung im Vergleich zu 752 $€$ für die ambulante Therapie. Nach einem Jahr summierten sich diese Beträge auf $1.272 €$ versus $1.148 €$. Wurden diese Kosten in Verhältnis zum Nutzen
(Anzahl der Tage mit einer Verbesserung des Hautzustandes um mindestens 50\%) gesetzt, erwies sich die Heim-Therapie als etwas effektiver, aber auch geringfügig teurer als die ambulante Therapie. Die Unterschiede waren nicht signifikant.

Fazit: Die Autoren empfehlen die Heimbehandlung als kosteneffektive Alternative zur ambulanten Therapie. Angesichts der hohen Patientenzufriedenheit ist der Heimbehandlung eindeutig der Vorzug zu geben.

Koek MB et al. Cost effectiveness of home ultraviolet $B$ phototherapy for psoriasis: economic evaluation of a randomised controlled trial (PLUTO study). BMJ 2010; 340: c1490. 